\title{
Intuitionistic Fuzzy Soft Rough Set and Its Application in Decision Making
}

\author{
Haidong Zhang, ${ }^{1,2}$ Lan Shu, ${ }^{1}$ and Shilong Liao ${ }^{2}$ \\ ${ }^{1}$ School of Mathematical Sciences, University of Electronic Science and Technology of China, Chengdu, Sichuan 611731, China \\ ${ }^{2}$ School of Mathematics and Computer Science, Northwest University for Nationalities, Lanzhou, Gansu 730030, China \\ Correspondence should be addressed to Haidong Zhang; lingdianstar@163.com
}

Received 29 November 2013; Revised 31 March 2014; Accepted 7 April 2014; Published 7 May 2014

Academic Editor: Chuanzhi Bai

Copyright (c) 2014 Haidong Zhang et al. This is an open access article distributed under the Creative Commons Attribution License, which permits unrestricted use, distribution, and reproduction in any medium, provided the original work is properly cited.

\begin{abstract}
The soft set theory, originally proposed by Molodtsov, can be used as a general mathematical tool for dealing with uncertainty. In this paper, we present concepts of soft rough intuitionistic fuzzy sets and intuitionistic fuzzy soft rough sets, and investigate some properties of soft rough intuitionistic fuzzy sets and intuitionistic fuzzy soft rough sets in detail. Furthermore, classical representations of intuitionistic fuzzy soft rough approximation operators are presented. Finally, we develop an approach to intuitionistic fuzzy soft rough sets based on decision making and a numerical example is provided to illustrate the developed approach.
\end{abstract}

\section{Introduction}

Many complicated problems in economics, engineering, social sciences, medical sciences, and many other fields involve uncertain data. These problems, which one comes face to face with in life, cannot be solved using classical mathematic methods. There are several well-known theories to describe uncertainty, for instance, fuzzy set theory [1], rough set theory $[2,3]$, and other mathematical tools. But all of these theories have their inherit difficulties as pointed out by Molodtsov [4]. To overcome these difficulties, in 1999 Molodtsov introduced the concept of soft sets, which can be seen as a new mathematical tool for dealing with uncertainties. This so-called soft set theory seems to be free from the difficulties affecting the existing methods. It has been found that fuzzy sets, rough sets, and soft sets are closely related concepts [5]. Soft set theory has potential applications in many different fields including the smoothness of functions, game theory, operational research, Perron integration, probability theory, and measurement theory $[4,6]$. Research works on soft sets are very active and progressing rapidly in these years. Maji et al. [7] defined several operations on soft sets and made a theoretical study on the theory of soft sets. Jun [8] introduced the notion of soft BCK/BCI-algebras.
Jun and Park [9] discussed the applications of soft sets in ideal theory of BCK/BCI-algebras. Feng et al. [10] applied soft set theory to the study of semirings and initiated the notion of soft semirings. Furthermore, based on [7], Ali et al. [11] introduced some new operations on soft sets and improved the notion of complement of soft set. They proved that certain De Morgan's laws hold in soft set theory. Qin and Hong [12] introduced the notion of soft equality and established lattice structures and soft quotient algebras of soft sets. Park et al. [13] discussed some properties of equivalence soft set relations.

The study of hybrid models combining soft sets with other mathematical structures is emerging as an active research topic of soft set theory. Maji et al. [14] initiated the study on hybrid structures involving fuzzy sets and soft sets. They introduced the notion of fuzzy soft sets, which can be seen as a fuzzy generalization of soft sets. Furthermore, based on [14], Majumdar and Samanta [15] modified the definition of fuzzy soft sets and presented the notion of generalized fuzzy soft sets theory. Yang et al. [16] presented the concept of the intervalvalued fuzzy soft sets by combining interval-valued fuzzy set $[17,18]$ and soft set models. By combining the multifuzzy set and soft set models, Yang et al. [19] presented the concept 
of the multifuzzy soft set and provided its application in decision making under an imprecise environment.

The concept of rough sets, proposed by Pawlak $[2,3]$ as a framework for the construction of approximations of concepts, is a formal tool for modeling and processing insufficient and incomplete information. Rough set theory is based on an assumption that every object in the universe of discourse is associated with some information. Objects characterized by the same information are indiscernible. The indiscernibility relation generated in this way forms the mathematical basis of the rough set theory. In general, the indiscernibility relation is also called equivalence relation. Then any subset of a universe can be characterized by two definable or observable subsets called lower and upper approximations. However, the equivalence relations in Pawlak rough set are too restrictive for many practical applications. In recent years, from both theoretical and practical needs, many authors have generalized the notion of Pawlak rough set by using nonequivalence binary relations. This has led to various other generalized rough set models [20-37]. More recently, rough set approximations have also been developed into the intuitionistic fuzzy environment, and the results are called intuitionistic fuzzy rough sets $[37,38]$, rough intuitionistic fuzzy sets [38], and generalized intuitionistic fuzzy rough sets $[39,40]$. By employing a special type of intuitionistic fuzzy triangular norm min, Zhou et al. [40] investigated a general framework for studying various relation-based intuitionistic fuzzy rough approximation operators in the constructive and axiomatic approaches. Zhou and $\mathrm{Wu}$ [41], based on intuitionistic fuzzy implicator, studied intuitionistic fuzzy rough approximations on one universe. Moreover, many new rough set models have also been established by combining the Pawlak rough set with other uncertainty theories such as soft set theory. Feng et al. [42] provided a framework to combine fuzzy sets, rough sets, and soft sets all together, which gives rise to several interesting new concepts such as rough soft sets, soft rough sets, and soft rough fuzzy sets. The combination of soft set and rough set models was also discussed by some researchers [43-45].

In this paper, we offer the notions of soft rough intuitionistic fuzzy sets and intuitionistic fuzzy soft rough sets, which can be seen as two new generalized soft rough set models. In order to give a new approach to decision making problems, we combine a fuzzy soft relation with intuitionistic fuzzy rough sets and propose the concept of intuitionistic fuzzy soft rough sets which is an extension of soft rough intuitionistic fuzzy sets. Then we can define the upper and lower approximations of any intuitionistic fuzzy set on parameter set $E$. Like the traditional intuitionistic fuzzy rough set models, intuitionistic fuzzy soft rough sets can also be exploited to extend many practical applications in reality. Therefore, we propose a novel approach to decision making based on intuitionistic fuzzy soft rough set theory.

The rest of this paper is organized as follows. In Section 2, we review some basic notions related to soft sets, fuzzy soft sets, and intuitionistic fuzzy sets. In Section 3, we construct the crisp soft rough approximation operators and discuss some of their interesting properties. By combining crisp soft relation with intuitionistic fuzzy sets, then the concept of soft rough intuitionistic fuzzy approximation operators is presented in Section 4, and the properties of the lower and upper soft rough intuitionistic fuzzy approximation operators are examined. In Section 5, we present the definition of intuitionistic fuzzy soft rough approximation operators which is an extension of soft rough intuitionistic fuzzy approximation operators in Section 4 and investigate some of their interesting properties. Furthermore, classical representations of intuitionistic fuzzy soft rough approximation operators are presented. Section 6 is devoted to studying the application of intuitionistic fuzzy soft rough sets. Some conclusions and outlooks for further research are given in Section 7.

\section{Preliminaries}

The following definitions and preliminaries are required in the sequel of our work and hence presented in brief.

Definition 1 (see [46]). Let $L^{*}=\{(\mu, \nu) \in[0,1] \times[0,1] \mid$ $\mu+\nu \leq 1\}$ and denote $\left(\mu_{1}, \nu_{1}\right) \leq_{L^{*}}\left(\mu_{2}, v_{2}\right) \Leftrightarrow \mu_{1} \leq \mu_{2}$ and $v_{1} \geq v_{2}, \forall\left(\mu_{1}, v_{1}\right),\left(\mu_{2}, v_{2}\right) \in L^{*}$. Then the pair $\left(L^{*}, \leq_{L^{*}}\right)$ is called a complete lattice. The operators $\wedge$ and $\vee$ on $\left(L^{*}, \leq_{L^{*}}\right)$ are defined as follows, for $\left(\mu_{1}, v_{1}\right),\left(\mu_{2}, v_{2}\right) \in L^{*}$ :

$$
\begin{aligned}
& \left(\mu_{1}, v_{1}\right) \wedge\left(\mu_{2}, v_{2}\right)=\left(\min \left\{\mu_{1}, \mu_{2}\right\}, \max \left\{v_{1}, v_{2}\right\}\right), \\
& \left(\mu_{1}, v_{1}\right) \vee\left(\mu_{2}, v_{2}\right)=\left(\max \left\{\mu_{1}, \mu_{2}\right\}, \min \left\{v_{1}, v_{2}\right\}\right) .
\end{aligned}
$$

Obviously, a complete lattice on $L^{*}$ has the smallest element $0_{L^{*}}=(0,1)$ and the greatest element $1_{L^{*}}=(1,0)$. The definitions of fuzzy logical operators can be straightforwardly extended to the intuitionistic fuzzy case. The strict partial order $<_{L^{*}}$ is defined by

$$
\begin{gathered}
\left(\mu_{1}, v_{1}\right)<_{L^{*}}\left(\mu_{2}, v_{2}\right) \Longleftrightarrow\left(\mu_{1}, v_{1}\right) \leq_{L^{*}}\left(\mu_{2}, v_{2}\right), \\
\left(\mu_{1}, v_{1}\right) \geq_{L^{*}}\left(\mu_{2}, v_{2}\right) .
\end{gathered}
$$

Definition 2 (see $[47,48])$. Let a set $U$ be fixed. An intuitionistic fuzzy (IF, for short) set $A$ in $U$ is an object having the form

$$
A=\left\{\left\langle x, \mu_{A}(x), \gamma_{A}(x)\right\rangle \mid x \in U\right\},
$$

where $\mu_{A}: U \rightarrow[0,1]$ and $\gamma_{A}: U \rightarrow[0,1]$ satisfy $0 \leq$ $\mu_{A}(x)+\gamma_{A}(x) \leq 1$ for all $x \in U$ and $\mu_{A}(x)$ and $\gamma_{A}(x)$ are, respectively, called the degree of membership and the degree of nonmembership of the element $x \in U$ to $A$.

The family of all intuitionistic fuzzy subsets in $U$ is denoted by $\operatorname{IF}(U)$. The complement of an IF set $A$ is denoted by $\sim A=\left\{\left\langle x, \gamma_{A}(x), \mu_{A}(x)\right\rangle \mid x \in U\right\}$.

Obviously, every fuzzy set $A=\{\langle x, A(x)\rangle \mid x \in U\}=$ $\left\{\left\langle x, \mu_{A}(x)\right\rangle \mid x \in U\right\}$ can be identified with the IF set of the form $\left\{\left\langle x, \mu_{A}(x), 1-\mu_{A}(x)\right\rangle \mid x \in U\right\}$ and is thus an IF set.

The basic operations on $\operatorname{IF}(U)$ are defined as follows [4751], for all $A, B \in \operatorname{IF}(U)$ :

(1) $A \subseteq B$ if and only if $\mu_{A}(x) \leq \mu_{B}(x)$ and $\gamma_{A}(x) \geq \gamma_{B}(x)$ for all $x \in U$,

(2) $A=B$ if and only if $A \subseteq B$ and $B \subseteq A$, 
(3) $A \cap B=\left\{\left\langle x, \min \left\{\mu_{A}(x), \mu_{B}(x)\right\}, \max \left\{\gamma_{A}(x), \gamma_{B}(x)\right\}\right\rangle \mid\right.$ $x \in U\}$,

(4) $A \cup B=\left\{\left\langle x, \max \left\{\mu_{A}(x), \mu_{B}(x)\right\}, \min \left\{\gamma_{A}(x), \gamma_{B}(x)\right\}\right\rangle \mid\right.$ $x \in U\}$.

For $(\alpha, \beta) \in L^{*}, \widehat{(\alpha, \beta)}$ denotes a constant IF set: $\widehat{(\alpha, \beta)}(x)=\{\langle x, \alpha, \beta\rangle \mid x \in U\}$, where $\alpha, \beta \in[0,1]$ and $\alpha+\beta \leq$ 1. The IF universe set is $U=1_{U}=\widehat{(1,0)}=\{\langle x, 1,0\rangle \mid x \in U\}$ and the IF empty set is $\emptyset=0_{U}=\widehat{(0,1)}=\{\langle x, 0,1\rangle \mid x \in U\}$.

For any $y \in U$, IF sets $1_{y}$ and $1_{U-\{y\}}$ are, respectively, defined as follows, for $x \in U$ :

$$
\begin{aligned}
\mu_{1_{y}}(x) & =\left\{\begin{array}{ll}
1, & x=y, \\
0, & x \neq y,
\end{array} \quad \gamma_{1_{y}}(x)= \begin{cases}0, & x=y, \\
1, & x \neq y,\end{cases} \right. \\
\mu_{1_{U-\{y\}}}(x) & =\left\{\begin{array}{ll}
0, & x=y, \\
1, & x \neq y,
\end{array} \quad \gamma_{1_{U-\{y\}}}(x)= \begin{cases}1, & x=y, \\
0, & x \neq y .\end{cases} \right.
\end{aligned}
$$

Definition 3 (see [38, 41]). Let $A=\left\{\left\langle x, \mu_{A}(x), \gamma_{A}(x)\right\rangle \mid x \in\right.$ $U\} \in \operatorname{IF}(U)$ and $(\alpha, \beta) \in L^{*}$. The $(\alpha, \beta)$-level cut set of $A$, denoted by $A_{\alpha}^{\beta}$, is defined as follows:

$$
A_{\alpha}^{\beta}=\left\{x \in U \mid \mu_{A}(x) \geq \alpha, \gamma_{A}(x) \leq \beta\right\} .
$$

$A_{\alpha}=\left\{x \in U \mid \mu_{A}(x) \geq \alpha\right\}$ and $A_{\alpha+}=\{x \in U \mid$ $\left.\mu_{A}(x)>\alpha\right\}$ are, respectively, called the $\alpha$-level cut set and the strong $\alpha$-level cut set of membership generated by $A$. And $A^{\beta}=\left\{x \in U \mid \gamma_{A}(x) \leq \beta\right\}$ and $A^{\beta+}=\left\{x \in U \mid \gamma_{A}(x)<\beta\right\}$ are, respectively, referred to as the $\beta$-level cut set and the strong $\beta$-level cut set of nonmembership generated by $A$.

At the same time, other types of cut sets of the IF set $A$ are denoted as follows:

$A_{\alpha+}^{\beta}=\left\{x \in U \mid \mu_{A}(x)>\alpha, \gamma_{A}(x) \leq \beta\right\}$, which is called the $(\alpha+, \beta)$-level cut set of $A$;

$A_{\alpha}^{\beta+}=\left\{x \in U \mid \mu_{A}(x) \geq \alpha, \gamma_{A}(x)<\beta\right\}$, which is called the $(\alpha, \beta+)$-level cut set of $A$;

$A_{\alpha+}^{\beta+}=\left\{x \in U \mid \mu_{A}(x)>\alpha, \gamma_{A}(x)<\beta\right\}$, which is called the $(\alpha+, \beta+)$-level cut set of $A$.

Theorem 4 (see $[38,41])$. The cut sets of IF sets satisfy the following properties, $\forall A \in \operatorname{IF}(U), \alpha, \beta \in[0,1]$ with $\alpha+\beta \leq 1$ :

(1) $A_{\alpha}^{\beta}=A_{\alpha} \cap A^{\beta}$,

(2) $(\sim A)_{\alpha}=\sim A^{\alpha+},(\sim A)^{\beta}=\sim A_{\beta+}$.

Definition 5 (see [4]). Let $U$ be an initial universe set and let $E$ be a universe set of parameters. A pair $(F, E)$ is called a soft set over $U$ if $F: E \rightarrow P(U)$, where $P(U)$ is the set of all subsets of $U$.

Definition 6 (see [52]). Let $(F, E)$ be a soft set over $U$. Then a subset of $U \times E$ called a crisp soft relation from $U$ to $E$ is uniquely defined by

$$
R=\left\{\left\langle(u, x), \mu_{R}(u, x)\right\rangle \mid(u, x) \in U \times E\right\},
$$

where $\mu_{R}: U \times E \rightarrow\{0,1\}, \mu_{R}(u, x)=\left\{\begin{array}{l}1,(u, x) \in R \\ 0,(u, x) \notin R\end{array}\right.$.
Definition 7 (see [14]). Let $U$ be an initial universe set and let $E$ be a universe set of parameters. A pair $(F, E)$ is called a fuzzy soft set over $U$ if $F: E \rightarrow F(U)$, where $F(U)$ is the set of all fuzzy subsets of $U$.

Definition 8 (see [53]). Let $(F, E)$ be a fuzzy soft set over $U$. Then a fuzzy subset of $U \times E$ called a fuzzy soft relation from $U$ to $E$ is uniquely defined by

$$
R=\left\{\left\langle(u, x), \mu_{R}(u, x)\right\rangle \mid(u, x) \in U \times E\right\},
$$

where $\mu_{R}: U \times E \rightarrow[0,1], \mu_{R}(u, x)=\mu_{F(x)}(u)$.

If $U=\left\{u_{1}, u_{2}, \ldots, u_{m}\right\}, E=\left\{x_{1}, x_{2}, \ldots, x_{n}\right\}$ then the fuzzy soft relation $R$ from $U$ to $E$ can be presented by a table as in the following form:

$$
\begin{array}{c|cccc}
R & x_{1} & x_{2} & \cdots & x_{n} \\
\hline u_{1} & \mu_{R}\left(u_{1}, x_{1}\right) & \mu_{R}\left(u_{1}, x_{2}\right) & \cdots & \mu_{R}\left(u_{1}, x_{n}\right) \\
u_{2} & \mu_{R}\left(u_{2}, x_{1}\right) & \mu_{R}\left(u_{2}, x_{2}\right) & \cdots & \mu_{R}\left(u_{2}, x_{n}\right) \\
\vdots & \vdots & \vdots & \ddots & \vdots \\
u_{m} & \mu_{R}\left(u_{m}, x_{1}\right) & \mu_{R}\left(u_{m}, x_{2}\right) & \cdots & \mu_{R}\left(u_{m}, x_{n}\right) .
\end{array}
$$

Definition 9 (see $[30,32])$. Let $U$ be a nonempty and finite universe of discourse and $R \subseteq U \times U$ an arbitrary crisp relation on $U$. We define a set-valued function $R_{s}: U \rightarrow P(U)$ by $R_{s}(x)=\{y \in U \mid(x, y) \in R\}, x \in U$.

The pair $(U, R)$ is called a crisp approximation space. For any $A \subseteq U$, the upper and lower approximations of $A$ with respect to $(U, R)$, denoted by $\bar{R}(A)$ and $\underline{R}(A)$, are defined, respectively, as follows:

$$
\begin{aligned}
& \bar{R}(A)=\left\{x \in U \mid R_{s}(x) \cap A \neq \emptyset\right\}, \\
& \underline{R}(A)=\left\{x \in U \mid R_{s}(x) \subseteq A\right\} .
\end{aligned}
$$

The pair $(\bar{R}(A), \underline{R}(A))$ is referred to as a crisp rough set, and $\bar{R}, \underline{R}: P(U) \rightarrow P(U)$ are, respectively, referred to as upper and lower crisp approximation operators induced from $(U, R)$.

\section{Construction of Soft Rough Sets}

In this section, by combining the crisp soft relation from $U$ to $E$ with crisp rough sets, we will introduce the concept of soft rough sets.

Definition 10. Let $U$ be an initial universe set and let $E$ be a universe set of parameters. For an arbitrary crisp soft relation $R$ over $U \times E$, we can define a set-valued function $R_{s}: U \rightarrow$ $P(E)$ by $R_{s}(u)=\{x \in E \mid(u, x) \in R\}, u \in U$.

$R$ is referred to as serial if for all $u \in U, R_{s}(u) \neq \emptyset$. The pair $(U, E, R)$ is called a crisp soft approximation space. For any $A \subseteq E$, the upper and lower soft approximations of $A$ with respect to $(U, E, R)$, denoted by $\bar{R}(A)$ and $\underline{R}(A)$, are defined, respectively, as follows:

$$
\begin{aligned}
& \bar{R}(A)=\left\{u \in U \mid R_{s}(u) \cap A \neq \emptyset\right\}, \\
& \underline{R}(A)=\left\{u \in U \mid R_{s}(u) \subseteq A\right\} .
\end{aligned}
$$


The pair $(\bar{R}(A), \underline{R}(A))$ is referred to as a crisp soft rough set, and $\bar{R}, \underline{R}: P(E) \rightarrow P(U)$ are referred to as upper and lower crisp soft rough approximation operators, respectively.

Example 11. Let $U$ be a universal set, which is denoted by $U=\left\{u_{1}, u_{2}, u_{3}, u_{4}, u_{5}\right\}$. Let $E$ be a set of parameters, where $E=\left\{e_{1}, e_{2}, e_{3}, e_{4}\right\}$. Suppose that a soft set over $U$ is defined as follows:

$$
\begin{gathered}
F\left(e_{1}\right)=\left\{u_{1}, u_{3}, u_{4}\right\}, \quad F\left(e_{2}\right)=\left\{u_{2}, u_{4}\right\}, \\
F\left(e_{3}\right)=\emptyset, \quad F\left(e_{4}\right)=U .
\end{gathered}
$$

Then the crisp soft relation on $U \times E$ is written by

$$
\begin{array}{r}
R=\left\{\left(u_{1}, e_{1}\right),\left(u_{3}, e_{1}\right),\left(u_{4}, e_{1}\right),\left(u_{2}, e_{2}\right),\left(u_{4}, e_{2}\right),\right. \\
\left.\left(u_{1}, e_{4}\right),\left(u_{2}, e_{4}\right),\left(u_{3}, e_{4}\right),\left(u_{4}, e_{4}\right),\left(u_{5}, e_{4}\right)\right\} .
\end{array}
$$

From Definition 10, we can obtain $R_{s}\left(u_{1}\right)=\left\{e_{1}, e_{4}\right\}$, $R_{s}\left(u_{2}\right)=\left\{e_{2}, e_{4}\right\}, R_{s}\left(u_{3}\right)=\left\{e_{1}, e_{4}\right\}, R_{s}\left(u_{4}\right)=\left\{e_{1}, e_{2}, e_{4}\right\}$, and $R_{s}\left(u_{5}\right)=\left\{e_{4}\right\}$.

If the set of parameter $A=\left\{e_{2}, e_{3}, e_{4}\right\}$, by (10) and (11), we have $\underline{R}(A)=\left\{u_{2}, u_{5}\right\}$ and $\bar{R}(A)=U$.

Theorem 12. Let $(U, E, R)$ be a crisp soft approximation space. Then upper and lower crisp soft approximation operators $\bar{R}(A)$ and $\underline{R}(A)$ in Definition 10 satisfy the following properties, for all $A, B \in P(E)$ :

$$
\begin{aligned}
& (C S L 1) \underline{R}(A)=\sim \bar{R}(\sim A), \\
& (C S U 1) \bar{R}(A)=\sim \underline{R}(\sim A), \\
& (C S L 2) \underline{R}(A \cap B)=\underline{R}(A) \cap \underline{R}(B), \\
& (C S U 2) \bar{R}(A \cup B)=\bar{R}(A) \cup \bar{R}(B), \\
& (C S L 3) A \subseteq B \Rightarrow \underline{R}(A) \subseteq \underline{R}(B), \\
& (C S U 3) A \subseteq B \Rightarrow \bar{R}(A) \subseteq \bar{R}(B), \\
& (C S L 4) \underline{R}(A \cup B) \supseteq \underline{R}(A) \cup \underline{R}(B), \\
& (C S U 4) \bar{R}(A \cap B) \subseteq \bar{R}(A) \cap \bar{R}(B),
\end{aligned}
$$

where $\sim A$ is the complement of $A$.

Proof. The proof can be directly obtained from Definition 10.

Properties (CSL1) and (CSU1) show that $\bar{R}$ and $\underline{R}$ are dual approximation operators. To illustrate the point, we introduce the following example.

Example 13. Reconsider Example 11; we have $\sim A=\left\{e_{1}\right\}$. By virtue of (10) and (11), we can obtain $\bar{R}(\sim A)=\left\{u_{1}, u_{3}, u_{4}\right\}$. It follows that $\sim \bar{R}(\sim A)=\left\{u_{2}, u_{5}\right\}$, which implies that (CSL1) holds; that is, $\underline{R}(A)=\sim \bar{R}(\sim A)$.

Similarly, we can verify that (CSU1) also holds.

Let another parameter set $B=\left\{e_{1}, e_{2}\right\}$; then we have $A \cup$ $B=\left\{e_{1}, e_{2}, e_{3}, e_{4}\right\}=E$ and $A \cap B=e_{2}$. From (10) and (11), we conclude that $\underline{R}(B)=\emptyset, \bar{R}(B)=\left\{u_{1}, u_{2}, u_{3}, u_{4}\right\}, \underline{R}(A \cap B)=\emptyset$, and $\bar{R}(A \cup B)=U$; that is, $\bar{R}(E)=U$.

Hence, (CSL2) and (CSU2) hold.
From Definition 10, the following theorem can be easily derived.

Theorem 14. Let $(U, E, R)$ be a crisp soft approximation space, and let $\bar{R}(A)$ and $\underline{R}(A)$ be the upper and lower soft approximations operators in Definition 10. Then,

$$
\begin{aligned}
R \text { is serial } & \Longleftrightarrow \underline{R}(A) \subseteq \bar{R}(A), \quad \forall A \subseteq E \\
& \Longleftrightarrow \underline{R}(\emptyset)=\emptyset \Longleftrightarrow \bar{R}(E)=U .
\end{aligned}
$$

Example 15. Reconsider Examples 11 and 13. It is noted that $R$ is serial. Then we have $\underline{R}(A) \subseteq \bar{R}(A)$. From Example 13, obviously $\underline{R}(\emptyset)=\emptyset$ and $\bar{R}(\bar{E})=U$ hold.

\section{Construction of Soft Rough Intuitionistic Fuzzy Sets}

Inspire by the concept of rough intuitionistic fuzzy sets in [38], we will present the concept of soft rough intuitionistic fuzzy sets by combining the crisp soft relation from $U$ to $E$ with the rough intuitionistic fuzzy sets and investigate the properties of soft rough intuitionistic fuzzy approximation operators.

Definition 16. Let $(U, E, R)$ be a crisp soft approximation space. For any $A=\left\{\left\langle x, \mu_{A}(x), \gamma_{A}(x)\right\rangle \mid x \in E\right\} \in \operatorname{IF}(E)$, the upper and lower soft approximations of $A$ with respect to $(U, E, R)$, denoted by $\bar{R}(A)$ and $\underline{R}(A)$, are, respectively, defined as follows:

$$
\begin{aligned}
& \bar{R}(A)=\left\{\left\langle u, \mu_{\bar{R}(A)}(u), \gamma_{\bar{R}(A)}(u)\right\rangle \mid u \in U\right\}, \\
& \underline{R}(A)=\left\{\left\langle u, \mu_{\underline{R}(A)}(u), \gamma_{\underline{R}(A)}(u)\right\rangle \mid u \in U\right\},
\end{aligned}
$$

where

$$
\begin{array}{ll}
\mu_{\bar{R}(A)}(u)=\bigvee_{x \in R_{s}(u)} \mu_{A}(x), & \gamma_{\bar{R}(A)}(u)=\bigwedge_{x \in R_{s}(u)} \gamma_{A}(x) ; \\
\mu_{\underline{R}(A)}(u)=\bigwedge_{x \in R_{s}(u)} \mu_{A}(x), & \gamma_{\underline{R}(A)}(u)=\bigvee_{x \in R_{s}(u)} \gamma_{A}(x) .
\end{array}
$$

We can note that $\bar{R}(A)$ and $\underline{R}(A)$ are two IF sets on $U$. Thus the pair $(\bar{R}(A), \underline{R}(A))$ is referred to as a soft rough IF set of $A$ with respect to $(U, E, R)$, and $\bar{R}, \underline{R}: \operatorname{IF}(E) \rightarrow \operatorname{IF}(U)$ are referred to as upper and lower soft rough IF approximation operators, respectively.

Remark 17. Let $(U, E, R)$ be a crisp soft approximation space. If $A=\left\{\left\langle x, \mu_{A}(x)\right\rangle \mid x \in U\right\} \in F(E)$, then the above soft rough IF approximation operators $\bar{R}(A)$ and $\underline{R}(A)$ degenerate to the following forms:

$$
\begin{aligned}
& \bar{R}(A)=\left\{\left\langle u, \mu_{\bar{R}(A)}(u)\right\rangle \mid u \in U\right\}, \\
& \underline{R}(A)=\left\{\left\langle u, \mu_{\underline{R}(A)}(u)\right\rangle \mid u \in U\right\},
\end{aligned}
$$


where $\mu_{\bar{R}(A)}(u)=\bigvee_{x \in R_{s}(u)} \mu_{A}(x)$ and $\mu_{\underline{R}(A)}(u)=$ $\bigwedge_{x \in R_{s}(u)} \mu_{A}(x)$.

In this case, we call the pair $(\bar{R}(A), \underline{R}(A))$ soft rough fuzzy set. This conclusion is similar to the traditional rough fuzzy set.

Remark 18. Let $(U, E, R)$ be a crisp soft approximation space. If $A \in P(E)$ is a crisp set of $E$, then the above soft rough IF approximation operators $\bar{R}(A)$ and $\underline{R}(A)$ degenerate to crisp soft rough approximation operators defined by Definition 10. Hence, soft rough IF approximation operators in Definition 16 are an extension of crisp soft rough approximation operators defined by Definition 10.

Example 19. Consider Example 11. Suppose the crisp soft relation on $U \times E$

$$
\begin{array}{r}
R=\left\{\left(u_{1}, e_{1}\right),\left(u_{3}, e_{1}\right),\left(u_{4}, e_{1}\right),\left(u_{2}, e_{2}\right),\left(u_{4}, e_{2}\right),\right. \\
\left.\left(u_{1}, e_{4}\right),\left(u_{2}, e_{4}\right),\left(u_{3}, e_{4}\right),\left(u_{4}, e_{4}\right),\left(u_{5}, e_{4}\right)\right\} .
\end{array}
$$

We can define an IF set $A \in \operatorname{IF}(E)$ as follows:

$$
\begin{gathered}
A=\left\{\left\langle e_{1}, 0.75,0.15\right\rangle,\left\langle e_{2}, 0.85,0.12\right\rangle,\right. \\
\left.\left\langle e_{3}, 0.2,0.6\right\rangle,\left\langle e_{4}, 0.8,0.2\right\rangle\right\} .
\end{gathered}
$$

Then by (15) and (16), we have

$$
\begin{array}{ll}
\mu_{\bar{R}(A)}\left(u_{1}\right)=0.80, & \gamma_{\bar{R}(A)}\left(u_{1}\right)=0.15, \\
\mu_{\bar{R}(A)}\left(u_{2}\right)=0.85, & \gamma_{\bar{R}(A)}\left(u_{2}\right)=0.12, \\
\mu_{\bar{R}(A)}\left(u_{3}\right)=0.80, & \gamma_{\bar{R}(A)}\left(u_{3}\right)=0.15, \\
\mu_{\bar{R}(A)}\left(u_{4}\right)=0.85, & \gamma_{\bar{R}(A)}\left(u_{4}\right)=0.12, \\
\mu_{\bar{R}(A)}\left(u_{5}\right)=0.80, & \gamma_{\bar{R}(A)}\left(u_{5}\right)=0.20 ; \\
\mu_{\underline{R}(A)}\left(u_{1}\right)=0.75, & \gamma_{\underline{R}(A)}\left(u_{1}\right)=0.20, \\
\mu_{\underline{R}(A)}\left(u_{2}\right)=0.80, & \gamma_{\underline{R}(A)}\left(u_{2}\right)=0.20, \\
\mu_{\underline{R}(A)}\left(u_{3}\right)=0.75, & \gamma_{\underline{R}(A)}\left(u_{3}\right)=0.20, \\
\mu_{\underline{R}(A)}\left(u_{4}\right)=0.75, & \gamma_{\underline{R}(A)}\left(u_{4}\right)=0.20, \\
\mu_{\underline{R}(A)}\left(u_{5}\right)=0.80, & \gamma_{\underline{R}(A)}\left(u_{5}\right)=0.20 .
\end{array}
$$

Thus,

$$
\begin{aligned}
\bar{R}(A)=\{ & \left\langle u_{1}, 0.80,0.15\right\rangle, \\
& \left\langle u_{2}, 0.85,0.12\right\rangle,\left\langle u_{3}, 0.80,0.15\right\rangle, \\
& \left.\left\langle u_{4}, 0.85,0.12\right\rangle,\left\langle u_{5}, 0.80,0.20\right\rangle\right\}, \\
\underline{R}(A)=\{ & \left\langle u_{1}, 0.75,0.20\right\rangle, \\
& \left\langle u_{2}, 0.80,0.20\right\rangle,\left\langle u_{3}, 0.75,0.20\right\rangle, \\
& \left.\left\langle u_{4}, 0.75,0.20\right\rangle,\left\langle u_{5}, 0.80,0.20\right\rangle\right\} .
\end{aligned}
$$

Theorem 20. Let $(U, E, R)$ be a crisp soft approximation space. Then the upper and lower soft rough IF approximation operators $\bar{R}(A)$ and $\underline{R}(A)$ in Definition 10 satisfy the following properties, $\forall A, B \in \overline{I F}(E), \forall \alpha, \beta \in[0,1]$ with $\alpha+\beta \leq 1$ :

$$
\begin{aligned}
& \text { (SIFL1) } \underline{R}(A)=\sim \bar{R}(\sim A), \\
& (\text { SIFL2) } \underline{R}(A \cap B)=\underline{R}(A) \cap \underline{R}(B), \\
& (\text { SIFL3) } A \subseteq B \Rightarrow \underline{R}(A) \subseteq \underline{R}(B), \\
& (\text { SIFL4) } \underline{R}(A \cup B) \supseteq \underline{R}(A) \cup \underline{R}(B), \\
& (\text { SIFU1) } \bar{R}(A)=\sim \underline{R}(\sim A) \\
& (\text { SIFU2) } \bar{R}(A \cup B)=\bar{R}(A) \cup \bar{R}(B), \\
& (\text { SIFU3) } A \subseteq B \Rightarrow \bar{R}(A) \subseteq \bar{R}(B) \\
& (\text { SIFU4) } \bar{R}(A \cap B) \subseteq \bar{R}(A) \cap \bar{R}(B),
\end{aligned}
$$

where $\sim A$ is the complement of $A$.

Proof. We only prove properties of the lower soft rough IF approximation operator $\underline{R}(A)$. The upper soft rough IF approximation operator $\bar{R}(A)$ can be proved similarly.

(SIFL1) By Definition 16, then we have

$$
\begin{aligned}
\sim & \underline{R}(\sim A) \\
& =\left\{\left\langle u, \gamma_{\underline{R}(\sim A)}(u), \mu_{\underline{R}(\sim A)}(u)\right\rangle \mid u \in U\right\} \\
& =\left\{\left\langle u, \bigvee_{x \in R_{s}(u)} \gamma_{(\sim A)}(x), \bigwedge_{x \in R_{s}(u)} \mu_{(\sim A)}(x)\right\rangle \mid u \in U\right\} \\
& =\left\{\left\langle u, \bigvee_{x \in R_{s}(u)} \mu_{A}(x), \bigwedge_{x \in R_{s}(u)} \gamma_{A}(x)\right\rangle \mid u \in U\right\} \\
& =\left\{\left\langle u, \mu_{\bar{R}(\sim A)}(u), \gamma_{\underline{R}(A)}(u)\right\rangle \mid u \in U\right\}=\bar{R}(A) .
\end{aligned}
$$

(SIFL2) By virtue of (16), then we have

$$
\begin{aligned}
\underline{R} & (A \cap B) \\
& =\left\{\left\langle u, \mu_{\underline{R}(A \cap B)}(u), \gamma_{\underline{R}(A \cap B)}(u)\right\rangle \mid u \in U\right\} \\
& =\left\{\left\langle u, \bigwedge_{x \in R_{s}(u)} \mu_{A \cap B}(x), \bigvee_{x \in R_{s}(u)} \gamma_{A \cap B}(x)\right\rangle \mid u \in U\right\} \\
& =\left\{\left\langleu, \bigwedge_{x \in R_{s}(u)}\left(\mu_{A}(x) \wedge \mu_{B}(x)\right),\right.\right.
\end{aligned}
$$




$$
\begin{aligned}
& \left.\left.\bigvee_{x \in R_{s}(u)} \gamma_{A}(x) \vee \gamma_{B}(x)\right\rangle \mid u \in U\right\} \\
& =\left\{\left\langleu, \mu_{\underline{R}(A)}(u) \wedge \mu_{\underline{R}(B)}(u),\right.\right. \\
& \left.\left.\gamma_{\underline{R}(A)}(u) \vee \gamma_{\underline{R}(B)}(u)\right\rangle \mid u \in U\right\} \\
& =\underline{R}(A) \cap \underline{R}(B) .
\end{aligned}
$$

(SIFL3) It can be easily verified by Definition 16.

(SIFL4) By (SIFL4), it is straightforward.

In Theorem 20, properties (SIFL1) and (SIFU1) show that the upper and lower soft rough IF approximation operators $\bar{R}$ and $\underline{R}$ are dual to each other. The following example can also verify the point.

Example 21. Reconsider Example 19; we have

$$
\begin{gathered}
\sim A=\left\{\left\langle e_{1}, 0.15,0.75\right\rangle,\left\langle e_{2}, 0.12,0.85\right\rangle,\right. \\
\left.\left\langle e_{3}, 0.6,0.2\right\rangle,\left\langle e_{4}, 0.2,0.8\right\rangle\right\} .
\end{gathered}
$$

Similar to Example 19, we can obtain

$$
\begin{aligned}
\bar{R}(\sim A)=\{ & \left\langle u_{1}, 0.20,0.75\right\rangle, \\
& \left\langle u_{2}, 0.20,0.80\right\rangle,\left\langle u_{3}, 0.20,0.75\right\rangle, \\
& \left.\left\langle u_{4}, 0.20,0.75\right\rangle,\left\langle u_{5}, 0.20,0.80\right\rangle\right\} .
\end{aligned}
$$

It follows that

$$
\begin{aligned}
\sim \bar{R}(\sim A)=\{ & \left\langle u_{1}, 0.75,0.20\right\rangle, \\
& \left\langle u_{2}, 0.80,0.20\right\rangle,\left\langle u_{3}, 0.75,0.20\right\rangle, \\
& \left.\left\langle u_{4}, 0.75,0.20\right\rangle,\left\langle u_{5}, 0.80,0.20\right\rangle\right\},
\end{aligned}
$$

then we have

$$
\begin{array}{r}
A \cap B=\left\{\left\langle e_{1}, 0.75,0.15\right\rangle,\left\langle e_{2}, 0.50,0.30\right\rangle,\right. \\
\left.\left\langle e_{3}, 0.10,0.60\right\rangle,\left\langle e_{4}, 0.65,0.20\right\rangle\right\} .
\end{array}
$$

From (15) and (16), we conclude that

$$
\begin{aligned}
\underline{R}(B)=\{ & \left\langle u_{1}, 0.65,0.20\right\rangle, \\
& \left\langle u_{2}, 0.50,0.30\right\rangle,\left\langle u_{3}, 0.65,0.20\right\rangle, \\
& \left.\left\langle u_{4}, 0.50,0.30\right\rangle,\left\langle u_{5}, 0.65,0.20\right\rangle\right\},
\end{aligned}
$$

$$
\begin{aligned}
\underline{R}(A \cap B)=\{ & \left\langle u_{1}, 0.65,0.20\right\rangle, \\
& \left\langle u_{2}, 0.50,0.30\right\rangle,\left\langle u_{3}, 0.65,0.20\right\rangle, \\
& \left.\left\langle u_{4}, 0.50,0.30\right\rangle,\left\langle u_{5}, 0.65,0.20\right\rangle\right\} .
\end{aligned}
$$

Hence, (SIFL2) holds. Similarly, we can verify that (SIFU2) also holds.

\section{Construction of Intuitionistic Fuzzy Soft Rough Sets}

In [38], Zhou and Wu first presented the concept of intuitionistic fuzzy rough sets. In this section, by combining the fuzzy soft relation with the intuitionistic fuzzy rough sets [38], we introduce the constructive definition of intuitionistic fuzzy soft rough sets, further extend soft rough intuitionistic fuzzy sets in Section 4, and investigate some properties of intuitionistic fuzzy soft rough approximation operators.

Definition 22. Let $U$ be an initial universe set and let $E$ be a universe set of parameters. For an arbitrary fuzzy soft relation $R$ over $U \times E$, the pair $(U, E, R)$ is called a fuzzy soft approximation space. For any $A \in \operatorname{IF}(E)$, we define the upper and lower soft approximations of $A$ with respect to $(U, E, R)$, denoted by $\bar{R}(A)$ and $\underline{R}(A)$, respectively, as follows:

$$
\begin{aligned}
& \bar{R}(A)=\left\{\left\langle u, \mu_{\bar{R}(A)}(u), \gamma_{\bar{R}(A)}(u)\right\rangle \mid u \in U\right\}, \\
& \underline{R}(A)=\left\{\left\langle u, \mu_{\underline{R}(A)}(u), \gamma_{\underline{R}(A)}(u)\right\rangle \mid u \in U\right\},
\end{aligned}
$$

where

$$
\begin{aligned}
& \mu_{\bar{R}(A)}(u)=\bigvee_{x \in E}\left[\mu_{R}(u, x) \wedge \mu_{A}(x)\right], \\
& \gamma_{\bar{R}(A)}(u)=\bigwedge_{x \in E}\left[\left(1-\mu_{R}(u, x)\right) \vee \gamma_{A}(x)\right] ; \\
& \mu_{\underline{R}(A)}(u)=\bigwedge_{x \in E}\left[\left(1-\mu_{R}(u, x)\right) \vee \mu_{A}(x)\right], \\
& \gamma_{\underline{R}(A)}(u)=\bigvee_{x \in E}\left[\mu_{R}(u, x) \wedge \gamma_{A}(x)\right] .
\end{aligned}
$$

The pair $(\bar{R}(A), \underline{R}(A))$ is referred to as an IF soft rough set of $A$ with respect to $(U, E, R)$.

It can be easily verified that $\bar{R}(A)$ and $\underline{R}(A) \in \operatorname{IF}(U)$. In fact,

$$
\begin{aligned}
\mu_{\bar{R}(A)}(u)+\gamma_{\bar{R}(A)}(u) \\
=\bigvee_{x \in E}\left[\mu_{R}(u, x) \wedge \mu_{A}(x)\right] \\
\quad+\bigwedge_{x \in E}\left[\left(1-\mu_{R}(u, x)\right) \vee \gamma_{A}(x)\right]
\end{aligned}
$$




$$
\begin{aligned}
= & \bigvee_{x \in E}\left[\mu_{R}(u, x) \wedge \mu_{A}(x)\right]+1 \\
& -\bigvee_{x \in E}\left[\mu_{R}(u, x) \wedge\left(1-\gamma_{A}(x)\right)\right] \\
\leq & \bigvee_{x \in E}\left[\mu_{R}(u, x) \wedge\left(1-\gamma_{A}(x)\right)\right]+1 \\
& -\bigvee_{x \in E}\left[\mu_{R}(u, x) \wedge\left(1-\gamma_{A}(x)\right)\right]=1 .
\end{aligned}
$$

Hence, $\bar{R}(A) \in \operatorname{IF}(U)$. Similarly, we can obtain $\underline{R}(A) \in \operatorname{IF}(U)$. So we call $\bar{R}, \underline{R}: \operatorname{IF}(E) \rightarrow \operatorname{IF}(U)$ the upper and lower IF soft rough approximation operators, respectively.

Remark 23. Let $A \in \operatorname{IF}(E)$. If $(U, E, R)$ is a crisp soft approximation space, then IF soft rough approximation operators $\bar{R}(A)$ and $\underline{R}(A)$ in Definition 22 degenerate to the following forms:

$$
\begin{aligned}
& \bar{R}(A)=\left\{\left\langle u, \mu_{\bar{R}(A)}(u), \gamma_{\bar{R}(A)}(u)\right\rangle \mid u \in U\right\}, \\
& \underline{R}(A)=\left\{\left\langle u, \mu_{\underline{R}(A)}(u), \gamma_{\underline{R}(A)}(u)\right\rangle \mid u \in U\right\},
\end{aligned}
$$

where

$$
\begin{array}{ll}
\mu_{\bar{R}(A)}(u)=\bigvee_{x \in R_{s}(u)} \mu_{A}(x), & \gamma_{\bar{R}(A)}(u)=\bigwedge_{x \in R_{s}(u)} \gamma_{A}(x) ; \\
\mu_{\underline{R}(A)}(u)=\bigwedge_{x \in R_{s}(u)} \mu_{A}(x), & \gamma_{\underline{R}(A)}(u)=\bigvee_{x \in R_{s}(u)} \gamma_{A}(x) .
\end{array}
$$

In this case, we can note that IF soft rough approximation operators $\bar{R}(A)$ and $R(A)$ are identical with soft rough IF approximation operators in Definition 16. That is, IF soft rough approximation operators in Definition 22 are an extension of soft rough IF approximation operators in Definition 16.

Remark 24. Let $(U, E, R)$ be a fuzzy soft approximation space. If $A \in F(E)$, then IF soft rough approximation operators $\bar{R}(A)$ and $\underline{R}(A)$ degenerate to the following forms:

$$
\begin{aligned}
& \bar{R}(A)=\left\{\left\langle u, \mu_{\bar{R}(A)}(u)\right\rangle \mid u \in U\right\}, \\
& \underline{R}(A)=\left\{\left\langle u, \mu_{\underline{R}(A)}(u)\right\rangle \mid u \in U\right\},
\end{aligned}
$$

where $\mu_{\bar{R}(A)}(u)=\bigvee_{x \in E}\left[\mu_{R}(u, x) \wedge \mu_{A}(x)\right], \mu_{R(A)}(u)=$ $\bigwedge_{x \in E}\left[\left(1-\mu_{R}(u, x)\right) \vee \mu_{A}(x)\right]$.

In this case, IF soft rough approximation operators $\bar{R}(A)$ and $\underline{R}(A)$ are identical with the soft fuzzy rough approximation operators defined by Sun and $\mathrm{Ma}$ [45]. That is, IF soft rough approximation operators in Definition 22 are an extension of the soft fuzzy rough approximation operators defined by Sun and Ma [45].
Remark 25. Let $(U, E, R)$ be a fuzzy soft approximation space. If $A \in P(E)$, then IF soft rough approximation operators $\bar{R}(A)$ and $\underline{R}(A)$ degenerate to the following forms:

$$
\begin{aligned}
& \bar{R}(A)=\left\{\left\langle u, \mu_{\bar{R}(A)}(u)\right\rangle \mid u \in U\right\}, \\
& \underline{R}(A)=\left\{\left\langle u, \mu_{\underline{R}(A)}(u)\right\rangle \mid u \in U\right\},
\end{aligned}
$$

where $\mu_{\bar{R}(A)}(u)=\bigvee_{x \in A} \mu_{R}(u, x), \mu_{\underline{R}(A)}(u)=\bigwedge_{x \notin A}[(1-$ $\left.\left.\mu_{R}(u, x)\right)\right]$.

Example 26. Suppose that $U=\left\{u_{1}, u_{2}, u_{3}, u_{4}, u_{5}\right\}$ is the set of five houses under consideration of a decision maker to purchase. Let $E$ be a parameter set, where $E=\left\{e_{1}, e_{2}, e_{3}, e_{4}\right\}=$ \{expensive; beautiful; size; location\}. Mr. X wants to buy the house which qualifies with the parameters of $E$ to the utmost extent from available houses in $U$. Assume that $\mathrm{Mr}$. $\mathrm{X}$ describes the "attractiveness of the houses" by constructing a fuzzy soft set $(F, E)$ which is a fuzzy soft relation $R$ from $U$ to $E$. And it is presented by a table as in the following form:

\begin{tabular}{c|cccc}
$R$ & $e_{1}$ & $e_{2}$ & $e_{3}$ & $e_{4}$ \\
\hline$u_{1}$ & 0.7 & 0.1 & 0.5 & 0.3 \\
$u_{2}$ & 0.2 & 0.5 & 0.8 & 0.7 \\
$u_{3}$ & 0.3 & 0.3 & 0.1 & 0.6 \\
$u_{4}$ & 0.5 & 0.5 & 0.2 & 0.4 \\
$u_{5}$ & 0.5 & 0.4 & 0.1 & 0.8.
\end{tabular}

Now suppose that Mr. X gives the optimum normal decision object $A$ which is an IF subset defined as follows:

$$
\begin{array}{r}
A=\left\{\left\langle e_{1}, 0.6,0.3\right\rangle,\left\langle e_{2}, 0.4,0.6\right\rangle,\right. \\
\left.\left\langle e_{3}, 0.1,0.8\right\rangle,\left\langle e_{4}, 0.7,0.2\right\rangle\right\} .
\end{array}
$$

By virtue of (31) and (32), we have

$$
\begin{array}{ll}
\mu_{\bar{R}(A)}\left(u_{1}\right)=0.6, & \gamma_{\bar{R}(A)}\left(u_{1}\right)=0.3, \\
\mu_{\bar{R}(A)}\left(u_{2}\right)=0.7, & \gamma_{\bar{R}(A)}\left(u_{2}\right)=0.3, \\
\mu_{\bar{R}(A)}\left(u_{3}\right)=0.6, & \gamma_{\bar{R}(A)}\left(u_{3}\right)=0.4, \\
\mu_{\bar{R}(A)}\left(u_{4}\right)=0.5, & \gamma_{\bar{R}(A)}\left(u_{4}\right)=0.5, \\
\mu_{\bar{R}(A)}\left(u_{5}\right)=0.7, & \gamma_{\bar{R}(A)}\left(u_{5}\right)=0.2 ; \\
\mu_{\underline{R}(A)}\left(u_{1}\right)=0.5, & \gamma_{\underline{R}(A)}\left(u_{1}\right)=0.5, \\
\mu_{\underline{R}(A)}\left(u_{2}\right)=0.2, & \gamma_{\underline{R}(A)}\left(u_{2}\right)=0.8, \\
\mu_{\underline{R}(A)}\left(u_{3}\right)=0.7, & \gamma_{\underline{R}(A)}\left(u_{3}\right)=0.3, \\
\mu_{\underline{R}(A)}\left(u_{4}\right)=0.5, & \gamma_{\underline{R}(A)}\left(u_{4}\right)=0.5, \\
\mu_{\underline{R}(A)}\left(u_{5}\right)=0.6, & \gamma_{\underline{R}(A)}\left(u_{5}\right)=0.4 .
\end{array}
$$


Thus,

$$
\begin{aligned}
\bar{R}(A)= & \left\{u_{1}, 0.6,0.3\right\rangle, \\
& \left\langle u_{2}, 0.7,0.3\right\rangle,\left\langle u_{3}, 0.6,0.4\right\rangle, \\
& \left.\left\langle u_{4}, 0.5,0.5\right\rangle,\left\langle u_{5}, 0.7,0.2\right\rangle\right\}, \\
\underline{R}(A)=\{ & \left\langle u_{1}, 0.5,0.5\right\rangle, \\
& \left\langle u_{2}, 0.2,0.8\right\rangle,\left\langle u_{3}, 0.7,0.3\right\rangle, \\
& \left.\left\langle u_{4}, 0.5,0.5\right\rangle,\left\langle u_{5}, 0.6,0.4\right\rangle\right\} .
\end{aligned}
$$

Theorem 27. Let $(U, E, R)$ be a fuzzy soft approximation space. Then the upper and lower IF soft rough approximation operators $\bar{R}(A)$ and $\underline{R}(A)$ in Definition 16 satisfy the following properties, $\forall A, B \in \overline{I F}(E), \forall(\alpha, \beta) \in L^{*}$ :

$$
\begin{aligned}
& \text { (IFSL1) } \underline{R}(A)=\sim \bar{R}(\sim A), \\
& \text { (IFSL2) } \underline{R}(A \cap B)=\underline{R}(A) \cap \underline{R}(B), \\
& \text { (IFSL3) } A \subseteq B \Rightarrow \underline{R}(A) \subseteq \underline{R}(B), \\
& (I F S L 4) \underline{R}(A \cup B) \supseteq \underline{R}(A) \cup \underline{R}(B), \\
& (I F S U 1) \bar{R}(A)=\sim \underline{R}(\sim A), \\
& (I F S U 2) \bar{R}(A \cup B)=\bar{R}(A) \cup \bar{R}(B), \\
& (I F S U 3) A \subseteq B \Rightarrow \bar{R}(A) \subseteq \bar{R}(B), \\
& (I F S U 4) \bar{R}(A \cap B) \subseteq \bar{R}(A) \cap \bar{R}(B),
\end{aligned}
$$

where $\sim A$ is the complement of $A$.

Proof. It is similar to the proof of Theorem 20.

Example 28. Reconsider Example 26; we have

$$
\begin{aligned}
\sim A=\{ & \left\langle e_{1}, 0.3,0.6\right\rangle,\left\langle e_{2}, 0.6,0.4\right\rangle, \\
& \left.\left\langle e_{3}, 0.8,0.1\right\rangle,\left\langle e_{4}, 0.2,0.7\right\rangle\right\} .
\end{aligned}
$$

Similar to Example 26, we have

$$
\begin{aligned}
\bar{R}(\sim A)=\{ & \left\langle u_{1}, 0.5,0.5\right\rangle, \\
& \left\langle u_{2}, 0.8,0.2\right\rangle,\left\langle u_{3}, 0.3,0.7\right\rangle, \\
& \left.\left\langle u_{4}, 0.5,0.5\right\rangle,\left\langle u_{5}, 0.4,0.6\right\rangle\right\} .
\end{aligned}
$$

It follows that

$$
\begin{aligned}
\sim \bar{R}(\sim A)=\{ & \left\langle u_{1}, 0.5,0.5\right\rangle, \\
& \left\langle u_{2}, 0.2,0.8\right\rangle,\left\langle u_{3}, 0.7,0.3\right\rangle, \\
& \left.\left\langle u_{4}, 0.5,0.5\right\rangle,\left\langle u_{5}, 0.6,0.4\right\rangle\right\} .
\end{aligned}
$$

Thus (IFSL1) holds; that is, $\underline{R}(A)=\sim \bar{R}(\sim A)$.

Similarly, we can obtain $\bar{R}(A)=\sim \underline{R}(\sim A)$.

On the other hand, let

$$
\begin{array}{r}
B=\left\{\left\langle e_{1}, 0.5,0.2\right\rangle,\left\langle e_{2}, 0.3,0.5\right\rangle,\right. \\
\left.\left\langle e_{3}, 0.2,0.8\right\rangle,\left\langle e_{4}, 0.6,0.1\right\rangle\right\} .
\end{array}
$$

Then, we have

$$
\begin{array}{r}
A \cap B=\left\{\left\langle e_{1}, 0.5,0.3\right\rangle,\left\langle e_{2}, 0.3,0.6\right\rangle,\right. \\
\left.\left\langle e_{3}, 0.1,0.8\right\rangle,\left\langle e_{4}, 0.6,0.2\right\rangle\right\} .
\end{array}
$$

By virtue of (31) and (32), we have

$$
\begin{aligned}
\underline{R}(B)=\{ & \left\langle u_{1}, 0.5,0.5\right\rangle, \\
& \left\langle u_{2}, 0.2,0.8\right\rangle,\left\langle u_{3}, 0.6,0.3\right\rangle, \\
& \left.\left\langle u_{4}, 0.5,0.5\right\rangle,\left\langle u_{5}, 0.5,0.4\right\rangle\right\}, \\
\underline{R}(A \cap B)=\{ & \left\langle u_{1}, 0.5,0.5\right\rangle, \\
& \left\langle u_{2}, 0.2,0.8\right\rangle,\left\langle u_{3}, 0.6,0.3\right\rangle, \\
& \left.\left\langle u_{4}, 0.5,0.5\right\rangle,\left\langle u_{5}, 0.5,0.4\right\rangle\right\} .
\end{aligned}
$$

It follows that (IFSL2) holds. Similarly, we can verify that (IFSU2) also holds.

In Example 26, we can note that $\underline{R}(A) \nsubseteq \bar{R}(A)$. But if $R$ is referred to as a serial fuzzy soft relation from $U$ to parameter set $E$, that is, for each $u \in U$, there exists $e \in E$ such that $R(u, x)=1$, we have $\underline{R}(A) \subseteq \bar{R}(A)$. The following theorem will illustrate the result.

Theorem 29. Let $(U, E, R)$ be a fuzzy soft approximation space. If $R$ is serial, then the upper and lower IF soft rough approximation operators $\bar{R}(A)$ and $\underline{R}(A)$ in Definition 22 satisfy the following properties:

(1) $\underline{R}(\emptyset)=\emptyset, \bar{R}(E)=U$;

(2) $\underline{R}(A) \subseteq \bar{R}(A), \forall A \in I F(E)$.

Proof. It is straightforward by Definition 22.

Assume that $R$ is a fuzzy soft relation from $U$ to parameter set $E$; denote

$$
\begin{aligned}
R_{\alpha} & =\left\{(u, x) \mid \mu_{R}(u, x) \geq \alpha\right\}, \\
R_{\alpha}(u) & =\left\{x \in E \mid \mu_{R}(u, x) \geq \alpha\right\}, \\
R_{\alpha+} & =\left\{(u, x) \mid \mu_{R}(u, x)>\alpha\right\}, \\
R_{\alpha+}(u) & =\left\{x \in E \mid \mu_{R}(u, x)>\alpha\right\} .
\end{aligned}
$$

Then $R_{\alpha}$ and $R_{\alpha+}$ are two crisp soft relations on $U \times E$.

Theorems 30 and 31 show that the IF soft rough approximation operators can be represented by crisp soft rough approximation operators.

Theorem 30. Let $(U, E, R)$ be a fuzzy soft approximation space and $A \in I F(E)$. Then the upper IF rough approximation operator can be represented as follows, $\forall u \in U$ : 
(1)

$$
\begin{aligned}
\mu_{\bar{R}(A)}(u) & =\bigvee_{\alpha \in[0,1]}\left[\alpha \wedge \overline{R_{\alpha}}\left(A_{\alpha}\right)(u)\right] \\
& =\bigvee_{\alpha \in[0,1]}\left[\alpha \wedge \overline{R_{\alpha}}\left(A_{\alpha+}\right)(u)\right] \\
& =\bigvee_{\alpha \in[0,1]}\left[\alpha \wedge \overline{R_{\alpha+}}\left(A_{\alpha}\right)(u)\right] \\
& =\bigvee_{\alpha \in[0,1]}\left[\alpha \wedge \overline{R_{\alpha+}}\left(A_{\alpha+}\right)(u)\right] ;
\end{aligned}
$$

(2)

$$
\begin{aligned}
\gamma_{\bar{R}(A)}(u) & =\bigwedge_{\alpha \in[0,1]}\left[\alpha \vee\left(1-\overline{R_{1-\alpha}}\left(A^{\alpha}\right)(u)\right)\right] \\
& =\bigwedge_{\alpha \in[0,1]}\left[\alpha \vee\left(1-\overline{R_{1-\alpha}}\left(A^{\alpha+}\right)(u)\right)\right] \\
& =\bigwedge_{\alpha \in[0,1]}\left[\alpha \vee\left(1-\overline{R_{(1-\alpha)+}}\left(A^{\alpha}\right)(u)\right)\right] \\
& =\bigwedge_{\alpha \in[0,1]}\left[\alpha \vee\left(1-\overline{R_{(1-\alpha)+}}\left(A^{\alpha+}\right)(u)\right)\right] ;
\end{aligned}
$$

and moreover, for any $\alpha \in[0,1]$,

(3) $[\bar{R}(A)]_{\alpha+} \subseteq \overline{R_{\alpha+}}\left(A_{\alpha+}\right) \subseteq \overline{R_{\alpha+}}\left(A_{\alpha}\right) \subseteq \overline{R_{\alpha}}\left(A_{\alpha}\right) \subseteq$ $[\bar{R}(A)]_{\alpha}$

(4) $[\bar{R}(A)]^{\alpha+} \subseteq \overline{R_{(1-\alpha)+}}\left(A^{\alpha+}\right) \subseteq \overline{R_{(1-\alpha)+}}\left(A^{\alpha}\right) \subseteq$ $\overline{R_{1-\alpha}}\left(A^{\alpha}\right) \subseteq[\bar{R}(A)]^{\alpha}$.

Proof.

(1) For any $u \in U$, we have

$$
\begin{aligned}
& \bigvee_{\alpha \in[0,1]}\left[\alpha \wedge \overline{R_{\alpha}}\left(A_{\alpha}\right)(u)\right] \\
& =\sup \left\{\alpha \in[0,1] \mid u \in \overline{R_{\alpha}}\left(A_{\alpha}\right)\right\} \\
& =\sup \left\{\alpha \in[0,1] \mid R_{\alpha}(u) \cap A_{\alpha} \neq \emptyset\right\} \\
& =\sup \left\{\alpha \in[0,1] \mid \exists x \in E\left[x \in R_{\alpha}(u), x \in A_{\alpha}\right]\right\} \\
& =\sup \left\{\alpha \in[0,1] \mid \exists x \in E\left[\mu_{R}(u, x) \geq \alpha, \mu_{A}(x) \geq \alpha\right]\right\} \\
& =\bigvee_{x \in E}\left[\mu_{R}(u, x) \wedge \mu_{A}(x)\right]=\mu_{\bar{R}(A)}(u) .
\end{aligned}
$$

Similarly, we can prove

$$
\begin{aligned}
\mu_{\bar{R}(A)}(u) & =\bigvee_{\alpha \in[0,1]}\left[\alpha \wedge \overline{R_{\alpha}}\left(A_{\alpha+}\right)(u)\right] \\
& =\bigvee_{\alpha \in[0,1]}\left[\alpha \wedge \overline{R_{\alpha+}}\left(A_{\alpha}\right)(u)\right] \\
& =\bigvee_{\alpha \in[0,1]}\left[\alpha \wedge \overline{R_{\alpha+}}\left(A_{\alpha+}\right)(u)\right] .
\end{aligned}
$$

(2) By the definition of upper crisp soft rough approximation operator in Definition 10, we have

$$
\begin{aligned}
& \bigwedge_{\alpha \in[0,1]}\left[\alpha \vee\left(1-\overline{R_{1-\alpha}}\left(A^{\alpha}\right)(u)\right)\right] \\
& =\inf \left\{\alpha \in[0,1] \mid u \in \overline{R_{1-\alpha}}\left(A^{\alpha}\right)\right\} \\
& =\inf \left\{\alpha \in[0,1] \mid R_{1-\alpha}(u) \cap A^{\alpha} \neq \emptyset\right\} \\
& =\inf \left\{\alpha \in[0,1] \mid \exists x \in E\left[x \in R_{1-\alpha}(u), x \in A^{\alpha}\right]\right\} \\
& =\inf \left\{\alpha \in[0,1] \mid \exists x \in E\left[\mu_{R}(u, x) \geq 1-\alpha, \gamma_{A}(x) \leq \alpha\right]\right\} \\
& =\bigwedge_{x \in E}\left[\left(1-\mu_{R}(u, x)\right) \vee \gamma_{A}(x)\right]=\gamma_{\bar{R}(A)}(u) .
\end{aligned}
$$

Likewise, we can prove that

$$
\begin{aligned}
\gamma_{\bar{R}(A)}(u) & =\bigwedge_{\alpha \in[0,1]}\left[\alpha \vee\left(1-\overline{R_{1-\alpha}}\left(A^{\alpha+}\right)(u)\right)\right] \\
& =\bigwedge_{\alpha \in[0,1]}\left[\alpha \vee\left(1-\overline{R_{(1-\alpha)+}}\left(A^{\alpha}\right)(u)\right)\right] \\
& =\bigwedge_{\alpha \in[0,1]}\left[\alpha \vee\left(1-\overline{R_{(1-\alpha)+}}\left(A^{\alpha+}\right)(u)\right)\right] .
\end{aligned}
$$

(3) It is easily verified that $\overline{R_{\alpha+}}\left(A_{\alpha+}\right) \subseteq \overline{R_{\alpha+}}\left(A_{\alpha}\right) \subseteq$ $\overline{R_{\alpha}}\left(A_{\alpha}\right)$. We only need to prove that $[\bar{R}(A)]_{\alpha+} \subseteq \overline{R_{\alpha+}}\left(A_{\alpha+}\right)$ and $\overline{R_{\alpha}}\left(A_{\alpha}\right) \subseteq[\bar{R}(A)]_{\alpha}$.

In fact, $\forall u \in[\bar{R}(A)]_{\alpha+}$; we have $\mu_{\bar{R}(A)}(u)>\alpha$. According to Definition 22, $\bigvee_{x \in E}\left[\mu_{R}(u, x) \wedge \mu_{A}(x)\right]>\alpha$ holds. Then $\exists x_{0} \in E$, such that $\mu_{R}\left(u, x_{0}\right) \wedge \mu_{A}\left(x_{0}\right)>\alpha$; that is, $\mu_{R}\left(u, x_{0}\right)>$ $\alpha$ and $\mu_{A}\left(x_{0}\right)>\alpha$. Thus $x_{0} \in R_{\alpha+}(u)$ and $x_{0} \in A_{\alpha+}$. Consequently, $R_{\alpha+}(u) \cap A_{\alpha+} \neq \emptyset$. By Definition 10, we have $u \in \overline{R_{\alpha+}}\left(A_{\alpha+}\right)$. Hence $[\bar{R}(A)]_{\alpha+} \subseteq \overline{R_{\alpha+}}\left(A_{\alpha+}\right)$.

On the other hand, for any $u \in \overline{R_{\alpha}}\left(A_{\alpha}\right)$, we have $\overline{R_{\alpha}}\left(A_{\alpha}\right)(u)=1$. Since $\mu_{\bar{R}(A)}(u)=\bigvee_{\beta \in[0,1]}\left[\beta \wedge \overline{R_{\beta}}\left(A_{\beta}\right)(u)\right] \geq$ $\alpha \wedge \overline{R_{\alpha}}\left(A_{\alpha}\right)(u)=\alpha$, we obtain $u \in[\bar{R}(A)]_{\alpha}$. Hence, $\overline{R_{\alpha}}\left(A_{\alpha}\right) \subseteq$ $[\bar{R}(A)]_{\alpha}$.

(4) Similar to the proof of the above (3), it can easily be verified.

Theorem 31. Let $(U, E, R)$ be a fuzzy soft approximation space and $A \in I F(E)$. Then the lower IF rough approximation operator can be represented as follows, $\forall u \in U$ :

(1)

$$
\begin{aligned}
\mu_{\underline{R}(A)}(u) & =\bigwedge_{\alpha \in[0,1]}\left[\alpha \vee \underline{R_{1-\alpha}}\left(A_{\alpha+}\right)(u)\right] \\
& =\bigwedge_{\alpha \in[0,1]}\left[\alpha \vee \underline{R_{(1-\alpha)+}}\left(A_{\alpha}\right)(u)\right] \\
& =\bigwedge_{\alpha \in[0,1]}\left[\alpha \vee \underline{R_{(1-\alpha)+}}\left(A_{\alpha+}\right)(u)\right] \\
& =\bigwedge_{\alpha \in[0,1]}\left[\alpha \vee \underline{R_{1-\alpha}}\left(A_{\alpha}\right)(u)\right] ;
\end{aligned}
$$


(2)

$$
\begin{aligned}
\gamma_{\underline{R}(A)}(u) & =\bigvee_{\alpha \in[0,1]}\left[\alpha \wedge\left(1-\underline{R_{\alpha}}\left(A^{\alpha}\right)(u)\right)\right] \\
& =\bigvee_{\alpha \in[0,1]}\left[\alpha \wedge\left(1-\underline{R_{\alpha+}}\left(A^{\alpha}\right)(u)\right)\right] \\
& =\bigvee_{\alpha \in[0,1]}\left[\alpha \wedge\left(1-\underline{R_{\alpha+}}\left(A^{\alpha+}\right)(u)\right)\right] \\
& =\bigvee_{\alpha \in[0,1]}\left[\alpha \wedge\left(1-\underline{R_{\alpha}}\left(A^{\alpha+}\right)(u)\right)\right] ;
\end{aligned}
$$

and moreover, for any $\alpha \in[0,1]$,

(3) $[\underline{R}(A)]_{\alpha+} \subseteq \quad \underline{R_{1-\alpha}}\left(A_{\alpha+}\right) \subseteq \quad R_{(1-\alpha)+}\left(A_{\alpha+}\right) \subseteq$ $R_{(1-\alpha)+}\left(A_{\alpha}\right) \subseteq\left[\underline{R} \overline{R(A)}_{\alpha} ;\right.$

(4) $[\underline{R}(A)]^{\alpha+} \subseteq \underline{R_{\alpha}}\left(A^{\alpha+}\right) \subseteq \underline{R_{\alpha+}}\left(A^{\alpha+}\right) \subseteq \underline{R_{\alpha+}}\left(A^{\alpha}\right) \subseteq$ $[\underline{R}(A)]^{\alpha}$.

Proof. ((1) and (2)) According to Theorems 29 and 4, for all $u \in U$, we have

$$
\begin{aligned}
\mu_{\bar{R}(\sim A)}(u) & =\bigvee_{\alpha \in[0,1]}\left[\alpha \wedge \overline{R_{\alpha}}(\sim A)_{\alpha}(u)\right] \\
& =\bigvee_{\alpha \in[0,1]}\left[\alpha \wedge \overline{R_{\alpha}}\left(\sim A^{\alpha+}\right)(u)\right] \\
& =\bigvee_{\alpha \in[0,1]}\left[\alpha \wedge\left(\sim \underline{R_{\alpha}}\left(A^{\alpha+}\right)\right)(u)\right] \\
& =\bigvee_{\alpha \in[0,1]}\left[\alpha \wedge\left(1-\underline{R_{\alpha}}\left(A^{\alpha+}\right)(u)\right)\right], \\
\gamma_{\bar{R}(\sim A)}(u) & =\bigwedge_{\alpha \in[0,1]}\left[\alpha \vee\left(1-\overline{R_{1-\alpha}}(\sim A)^{\alpha}(u)\right)\right] \\
& =\bigwedge_{\alpha \in[0,1]}\left[\alpha \vee\left(1-\overline{R_{1-\alpha}}\left(\sim A_{\alpha+}\right)(u)\right)\right] \\
& =\bigwedge_{\alpha \in[0,1]}\left[\alpha \vee\left(1-\left(\sim \underline{R_{1-\alpha}}\left(A_{\alpha+}\right)\right)(u)\right)\right] \\
& =\bigwedge_{\alpha \in[0,1]}\left[\alpha \vee \underline{R_{1-\alpha}}\left(A_{\alpha+}\right)(u)\right] .
\end{aligned}
$$

Hence, by the duality of upper and lower IF soft rough approximation operators (see Theorem 27), we can conclude

$$
\begin{gathered}
\mu_{\underline{R}(A)}(u)=\gamma_{\bar{R}(\sim A)}(u)=\bigwedge_{\alpha \in[0,1]}\left[\alpha \vee \underline{R_{1-\alpha}}\left(A_{\alpha+}\right)(u)\right], \\
\gamma_{\underline{R}(A)}(u)=\mu_{\bar{R}(\sim A)}(u)=\bigvee_{\alpha \in[0,1]}\left[\alpha \wedge\left(1-\underline{R_{\alpha}}\left(A^{\alpha+}\right)(u)\right)\right] .
\end{gathered}
$$

Similar to the above proof, the others can be verified.

((3) and (4)) It is similar to the proof of Theorems 30 (3) and $30(4)$.

\section{Application of IF Soft Rough Sets in Decision Making}

In the above-mentioned sections, to demonstrate the validity of those new models' properties, several examples are carried out. For example, by data validation all upper and lower approximation operators $\bar{R}$ and $\underline{R}$ in the above examples are dual to each other and possess monotonicity. By those examples, the models are further understood, laying a good foundation for further study and application.

In this section, in order to illustrate the validity of IF soft rough sets in Section 5, we present an approach to the decision making based on IF soft rough sets.

Let $(U, E, R)$ be a fuzzy soft approximation space, where $U$ is the universe of the discourse, $E$ is the parameter set, and $R$ is a fuzzy soft relation on $U \times E$. Then we can give an algorithm based on IF soft rough sets with five steps.

First, according to their own needs, the decision makers can construct a fuzzy soft relation $R$ from $U$ to $E$, or fuzzy soft set $(F, E)$ over $U$.

Second, for a certain decision evaluation problem, each person has various opinions on the attributes of the same parameter. For example, in Example 26, Mr. X gives the optimum normal decision object $A$ defined by

$$
\begin{array}{r}
A=\left\{\left\langle e_{1}, 0.6,0.3\right\rangle,\left\langle e_{2}, 0.4,0.6\right\rangle,\right. \\
\left.\left\langle e_{3}, 0.1,0.8\right\rangle,\left\langle e_{4}, 0.7,0.2\right\rangle\right\} .
\end{array}
$$

That is to say, to the parameter element $e_{1}, \mathrm{Mr}$. X thinks that an expensive house is on the membership degree of 0.6 and is on the nonmembership degree of 0.3 . However, Mrs. X may not think it. Maybe she thinks that an expensive house is on the membership degree of 0.4 and is on the nonmembership degree of 0.5 .

So, we can construct an optimum normal decision object $A$ on the basis of assumption, according to different needs of the decision maker.

Third, by (31) and (32), we can compute the IF soft rough approximation operators $\bar{R}(A)$ and $\underline{R}(A)$ of the optimum normal decision object $A$. Thus, we obtain two most close values $\bar{R}(A)$ and $\underline{R}(A)$ to the decision alternative $u_{j}$ of the universe set $U$.

Fourth, $\mathrm{Xu}$ and Yager $[54,55]$ gave two operations on two IFs, shown as follows, for all $A, B \in \operatorname{IF}(U)$.

(i) Union operation:

$$
\begin{aligned}
A \cup B=\left\{\left\langlex, \max \left\{\mu_{A}(x), \mu_{B}(x)\right\},\right.\right. & \\
& \left.\left.\min \left\{\gamma_{A}(x), \gamma_{B}(x)\right\}\right\rangle \mid x \in U\right\} .
\end{aligned}
$$

(ii) Intersection operation:

$$
\begin{aligned}
A \cap B=\left\{\left\langlex, \min \left\{\mu_{A}(x), \mu_{B}(x)\right\},\right.\right. & \\
& \left.\left.\max \left\{\gamma_{A}(x), \gamma_{B}(x)\right\}\right\rangle \mid x \in U\right\} .
\end{aligned}
$$

(iii) Ring sum operation:

$$
\begin{gathered}
A \oplus B=\left\{\left\langlex, \mu_{A}(x)+\mu_{B}(x)-\mu_{A}(x) \mu_{B}(x),\right.\right. \\
\left.\left.\gamma_{A}(x) \gamma_{B}(x)\right\rangle \mid x \in U\right\} .
\end{gathered}
$$


(iv) Ring product operation:

$$
\begin{gathered}
A \otimes B=\left\{\left\langlex, \mu_{A}(x) \mu_{B}(x), \gamma_{A}(x)+\gamma_{B}(x)\right.\right. \\
\left.\left.-\gamma_{A}(x) \gamma_{B}(x)\right\rangle \mid x \in U\right\} .
\end{gathered}
$$

In general, the union operation and intersection operation can result in loss of information, thus affecting the accuracy of decision making that could lead to unreasonable decision. So we adopt the ring sum operation and ring product operation in decision making. As Xu $[54,55]$ pointed out, however, we are used to adopt the ring sum operation. Of course, adopting ring product operation is not wrong in decision making, which may result in the different decisions.

So, by the ring sum operation, we can compute the choice set

$$
\begin{aligned}
H= & \bar{R}(A) \oplus \underline{R}(A) \\
= & \left\{\left\langleu, \mu_{\bar{R}(A)}(u)+\mu_{\underline{R}(A)}(u)-\mu_{\bar{R}(A)}(u) \cdot \mu_{\underline{R}(A)}(u),\right.\right. \\
& \left.\left.\gamma_{\bar{R}(A)}(u) \cdot \gamma_{\underline{R}(A)}(u)\right\rangle \mid u \in U\right\} .
\end{aligned}
$$

Denote $H=\left\{\left\langle u, \mu_{H}(u), \gamma_{H}(u)\right\rangle\right\}$.

Finally, we should take the object $u_{j} \in U$ in universe $U$ with the maximum choice value as the optimum decision for the given decision making problem.

In order to do that, we construct a top-level threshold value $\lambda=(\mu, \gamma) \in L^{*}$, where $\mu=\max _{1 \leq i \leq n} \mu_{H}\left(u_{i}\right), \gamma=$ $\min _{1 \leq i \leq n} \gamma_{H}\left(u_{i}\right)$. Obviously, IF value $\lambda=(\mu, \gamma)$ is the maximum choice value in the choice set $H$. If $\mu_{H}\left(u_{j}\right) \geq_{L^{*}} \mu$ and $\gamma_{H}\left(u_{j}\right) \geq_{L^{*}} \gamma$, the optimum decision is $u_{j}$.

In the last step of the above algorithm, one may go back to the second step and change decision object so that the final decision is only one, when there exist too many "optimal choices" to be chosen.

To illustrate the idea of the algorithm given above, let us consider the example as follows.

Example 32. Reconsider Example 26. In Example 26, we have computed IF soft rough approximation operators $\bar{R}(A)$ and $\underline{R}(A)$ of the optimum normal decision object $A$. Now by using the fourth step of the algorithm for IF soft rough sets in decision making presented in this section, we can obtain

$$
\begin{aligned}
H= & \bar{R}(A) \oplus \underline{R}(A) \\
= & \left\{\left\langle u_{1}, 0.80,0.15\right\rangle,\left\langle u_{2}, 0.76,0.24\right\rangle,\left\langle u_{3}, 0.88,0.12\right\rangle,\right. \\
& \left.\left\langle u_{4}, 0.75,0.25\right\rangle,\left\langle u_{5}, 0.88,0.05\right\rangle\right\} .
\end{aligned}
$$

Obviously, the optimal decision is $u_{5}$.

On the other hand, by adopting the ring product operation, we have

$$
\begin{aligned}
H= & \bar{R}(A) \otimes \underline{R}(A) \\
= & \left\{\left\langle u_{1}, 0.3,0.65\right\rangle,\left\langle u_{2}, 0.14,0.86\right\rangle,\left\langle u_{3}, 0.42,0.58\right\rangle,\right. \\
& \left.\left\langle u_{4}, 0.25,0.75\right\rangle,\left\langle u_{5}, 0.42,0.52\right\rangle\right\} .
\end{aligned}
$$

It is noted that the optimal decision is still $u_{5}$. Hence, Mr. X will buy the house $u_{5}$.

\section{Conclusion}

In this paper, we have first presented two new concepts: soft rough intuitionistic fuzzy sets and intuitionistic fuzzy soft rough sets, and we investigated some properties of soft rough intuitionistic fuzzy sets and intuitionistic fuzzy soft rough sets in detail. In addition, a new decision method based on intuitionistic fuzzy soft rough sets is first proposed. Moreover, to illustrate the validity of the model, a practical application based on intuitionistic fuzzy soft rough sets is applied to show its validity. We also believe that the decision making method developed here is expected to attract the researchers working in these areas.

It is noted that the decision method in the last step needs to choose the thresholds in advance by decision makers. Then the results will depend on the threshold values to a certain extent. Since the thresholds are subjective, the different experts can obtain the different decision results for the same decision problem. So, in order to avoid the effect of the subjective information for the decision results, we use the data information provided by the decision making problem only and do not need any additional available information provided by decision makers. Thus the decision results are more objective.

Actually, there are at least two aspects in the study of rough set theory: constructive and axiomatic approaches, so is the same to intuitionistic fuzzy soft rough sets. So further work should consider the axiomatic approaches to intuitionistic fuzzy soft rough sets and the modification of the proposed decision method.

\section{Conflict of Interests}

The authors declare that there is no conflict of interests regarding the publication of this paper.

\section{Acknowledgments}

The authors would like to thank the anonymous referees for their valuable comments and suggestions. This work is supported by the Natural Science Foundation of China (no. 71261022) and the Fundamental Research Funds for the Central Universities of Northwest University for Nationalities (no. zyz2012076).

\section{References}

[1] L. A. Zadeh, "Fuzzy sets," Information and Control, vol. 8, no. 3, pp. 338-353, 1965.

[2] Z. Pawlak, "Rough sets," International Journal of Computer \& Information Sciences, vol. 11, no. 5, pp. 145-172, 1982.

[3] Z. Pawlak, Rough Sets-Theoretical Aspects to Reasoning about Data, Kluwer Academic Publisher, Boston, Mass, USA, 1991.

[4] D. Molodtsov, "Soft set theory-first results," Computers and Mathematics with Applications, vol. 37, no. 4-5, pp. 19-31, 1999. 
[5] H. Aktaş and N. Çağman, "Soft sets and soft groups," Information Sciences, vol. 177, no. 13, pp. 2726-2735, 2007.

[6] D. Molodtsov, The Theory of Soft Sets, URSS Publishers, Moscow, Russia, 2004 (Russian).

[7] P. K. Maji, R. Biswas, and A. R. Roy, "Soft set theory," Computers and Mathematics with Applications, vol. 45, no. 4-5, pp. 555-562, 2003.

[8] Y. B. Jun, "Soft BCK/BCI-algebras," Computers and Mathematics with Applications, vol. 56, no. 5, pp. 1408-1413, 2008.

[9] Y. B. Jun and C. H. Park, "Applications of soft sets in ideal theory of BCK/BCI-algebras," Information Sciences, vol. 178, no. 11, pp. 2466-2475, 2008.

[10] F. Feng, Y. B. Jun, and X. Zhao, "Soft semirings," Computers and Mathematics with Applications, vol. 56, no. 10, pp. 2621-2628, 2008.

[11] M. I. Ali, F. Feng, X. Liu, W. K. Min, and M. Shabir, "On some new operations in soft set theory," Computers and Mathematics with Applications, vol. 57, no. 9, pp. 1547-1553, 2009.

[12] K. Qin and Z. Hong, "On soft equality," Journal of Computational and Applied Mathematics, vol. 234, no. 5, pp. 1347-1355, 2010.

[13] J. H. Park, O. H. Kim, and Y. C. Kwun, "Some properties of equivalence soft set relations," Computers and Mathematics with Applications, vol. 63, no. 6, pp. 1079-1088, 2012.

[14] P. K. Maji, R. Biswas, and A. R. Roy, "Fuzzy soft set," Journal of Fuzzy Mathematics, vol. 9, no. 3, pp. 589-602, 2001.

[15] P. Majumdar and S. K. Samanta, "Generalised fuzzy soft sets," Computers and Mathematics with Applications, vol. 59, no. 4, pp. 1425-1432, 2010.

[16] X. Yang, T. Y. Lin, J. Yang, Y. Li, and D. Yu, "Combination of interval-valued fuzzy set and soft set," Computers and Mathematics with Applications, vol. 58, no. 3, pp. 521-527, 2009.

[17] M. B. Gorzałczany, "A method of inference in approximate reasoning based on interval-valued fuzzy sets," Fuzzy Sets and Systems, vol. 21, no. 1, pp. 1-17, 1987.

[18] G. Deschrijver and E. E. Kerre, "Implicators based on binary aggregation operators in interval-valued fuzzy set theory," Fuzzy Sets and Systems, vol. 153, no. 2, pp. 229-248, 2005.

[19] Y. Yang, X. Tan, and C. C. Meng, "The multi-fuzzy soft set and its application in decision making," Applied Mathematical Modelling, vol. 37, pp. 4915-4923, 2013.

[20] Y. Bi, S. McClean, and T. Anderson, "Combining rough decisions for intelligent text mining using Dempster's rule," Artificial Intelligence Review, vol. 26, no. 3, pp. 191-209, 2006.

[21] Z. Bonikowski, E. Bryniarski, and U. Wybraniec-Skardowska, "Extensions and intentions in the rough set theory," Information Sciences, vol. 107, no. 1-4, pp. 149-167, 1998.

[22] Z. Gong, B. Sun, and D. Chen, "Rough set theory for the interval-valued fuzzy information systems," Information Sciences, vol. 178, no. 8, pp. 1968-1985, 2008.

[23] J. S. Mi and W. X. Zhang, "An axiomatic characterization of a fuzzy generalization of rough sets," Information Sciences, vol. 160, no. 1-4, pp. 235-249, 2004.

[24] S. P. Tiwari and A. K. Srivastava, "Fuzzy rough sets, fuzzy preorders and fuzzy topologies," Fuzzy Sets and Systems, vol. 210, pp. 63-68, 2013.

[25] W. Z. Wu, Y. Leung, and W. X. Zhang, "On generalized rough fuzzy approximation operators," in Transactions on Rough Sets $V$, vol. 4100 of Lecture Notes in Computer Science, pp. 263-284, Springer, Berlin, Germany, 2006.
[26] W.-Z. Wu, Y. Leung, and J.-S. Mi, "On characterizations of $(\mathscr{I}, \mathscr{T})$-fuzzy rough approximation operators," Fuzzy Sets and Systems, vol. 154, no. 1, pp. 76-102, 2005.

[27] W. Z. Wu, Y. Leung, and W. X. Zhang, "Connections between rough set theory and Dempster-Shafer theory of evidence," International Journal of General Systems, vol. 31, no. 4, pp. 405430, 2002.

[28] W. Z. Wu, J. S. Mi, and W. X. Zhang, "Generalized fuzzy rough sets," Information Sciences, vol. 151, pp. 263-282, 2003.

[29] W. Z. Wu and W. X. Zhang, "Constructive and axiomatic approaches of fuzzy approximation operators," Information Sciences, vol. 159, no. 3-4, pp. 233-254, 2004.

[30] Y. Y. Yao, "Constructive and algebraic methods of the theory of rough sets," Information Sciences, vol. 109, no. 1-4, pp. 21-47, 1998.

[31] Y. Y. Yao, "Two views of the theory of rough sets in finite universes,' International Journal of Approximate Reasoning, vol. 15, no. 4, pp. 291-317, 1996.

[32] Y. Y. Yao, "Generalized rough set model," in Rough Sets in Knowledge Discovery 1: Methodology and Applications, L. Polkowski and A. Skowron, Eds., Studies in Fuzziness and Soft Computing, pp. 286-318, Physica, Berlin, Germany, 1998.

[33] Y. Y. Yao, "Relational interpretations of neighborhood operators and rough set approximation operators," Information Sciences, vol. 111, no. 1-4, pp. 239-259, 1998.

[34] M. I. Ali, B. Davvaz, and M. Shabir, "Some properties of generalized rough sets," Information Sciences, vol. 224, pp. 170179, 2013.

[35] Z. M. Zhang, "On characterization of generalized interval type2 fuzzy rough sets," Information Sciences, vol. 219, pp. 124-150, 2013.

[36] Z. Zhang, "On interval type-2 rough fuzzy sets," KnowledgeBased Systems, vol. 35, pp. 1-13, 2012.

[37] X. H. Zhang, B. Zhou, and P. Li, "A general frame for intuitionistic fuzzy rough sets," Information Sciences, vol. 216, pp. 34-49, 2012.

[38] L. Zhou and W. Z. Wu, "Characterization of rough set approximations in Atanassov intuitionistic fuzzy set theory," Computers and Mathematics with Applications, vol. 62, no. 1, pp. 282-296, 2011.

[39] Z. Zhang, "Generalized intuitionistic fuzzy rough sets based on intuitionistic fuzzy coverings," Information Sciences, vol. 198, pp. 186-206, 2012.

[40] L. Zhou, W. Z. Wu, and W. X. Zhang, "On characterization of intuitionistic fuzzy rough sets based on intuitionistic fuzzy implicators," Information Sciences, vol. 179, no. 7, pp. 883-898, 2009.

[41] L. Zhou and W.-Z. Wu, "On generalized intuitionistic fuzzy rough approximation operators," Information Sciences, vol. 178, no. 11, pp. 2448-2465, 2008.

[42] F. Feng, C. Li, B. Davvaz, and M. I. Ali, "Soft sets combined with fuzzy sets and rough sets: a tentative approach," Soft Computing, vol. 14, no. 9, pp. 899-911, 2010.

[43] M. Shabir, M. I. Ali, and T. Shaheen, "Another approach to soft rough sets," Knowledge-Based Systems, vol. 40, pp. 72-80, 2013.

[44] F. Feng, X. Liu, V. Leoreanu-Fotea, and Y. B. Jun, "Soft sets and soft rough sets," Information Sciences, vol. 181, no. 6, pp. 11251137, 2011.

[45] B. Sun and W. Ma, "Soft fuzzy rough sets and its application in decision making," Artificial Intelligence Review, vol. 41, pp. 6780, 2014. 
[46] C. Cornelis, G. Deschrijver, and E. E. Kerre, "Implication in intuitionistic fuzzy and interval-valued fuzzy set theory: construction, classification, application," International Journal of Approximate Reasoning, vol. 35, no. 1, pp. 55-95, 2004.

[47] K. T. Atanassov, "Intuitionistic fuzzy sets," Fuzzy Sets and Systems, vol. 20, no. 1, pp. 87-96, 1986.

[48] K. Atanassov, Intuitionistic Fuzzy Sets: Theory and Applications, Physica, Heidelberg, Germany, 1999.

[49] K. T. Atanassov, "More on intuitionistic fuzzy sets," Fuzzy Sets and Systems, vol. 33, no. 1, pp. 37-45, 1989.

[50] K. T. Atanassov, "New operations defined over the intuitionistic fuzzy sets," Fuzzy Sets and Systems, vol. 61, no. 2, pp. 137-142, 1994.

[51] K. T. Atanassov, "Remarks on the intuitionistic fuzzy sets-III," Fuzzy Sets and Systems, vol. 75, no. 3, pp. 401-402, 1995.

[52] N. Çağman and S. Enginoğlu, "Soft matrix theory and its decision making," Computers and Mathematics with Applications, vol. 59, no. 10, pp. 3308-3314, 2010.

[53] N. Cagman and S. Enginoglu, "Fuzzy soft matrix theory and its application in decision making," Iranian Journal of Fuzzy Systems, vol. 9, no. 1, pp. 109-119, 2012.

[54] Z. Xu, "Intuitionistic fuzzy aggregation operators," IEEE Transactions on Fuzzy Systems, vol. 15, no. 6, pp. 1179-1187, 2007.

[55] Z. Xu and R. R. Yager, "Some geometric aggregation operators based on intuitionistic fuzzy sets," International Journal of General Systems, vol. 35, no. 4, pp. 417-433, 2006. 


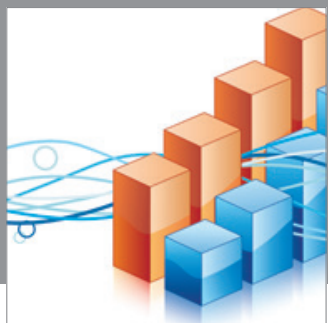

Advances in

Operations Research

mansans

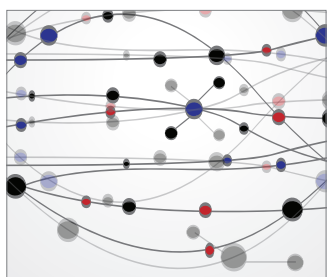

The Scientific World Journal
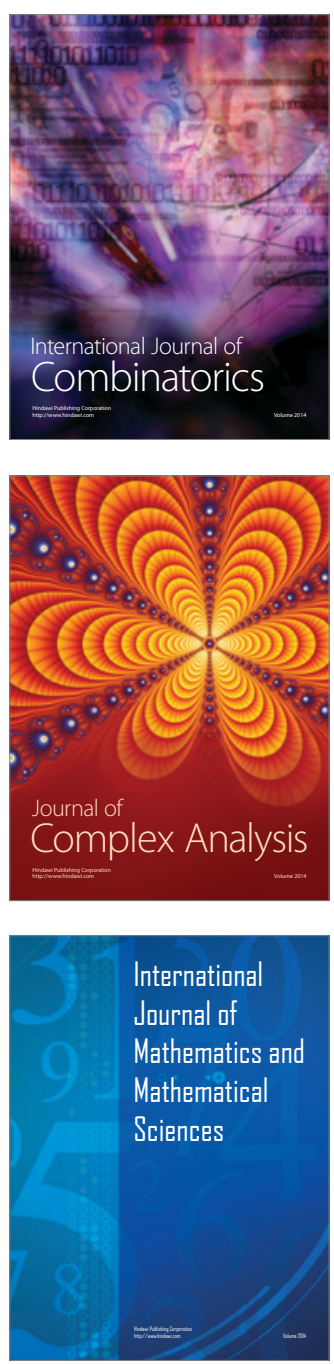
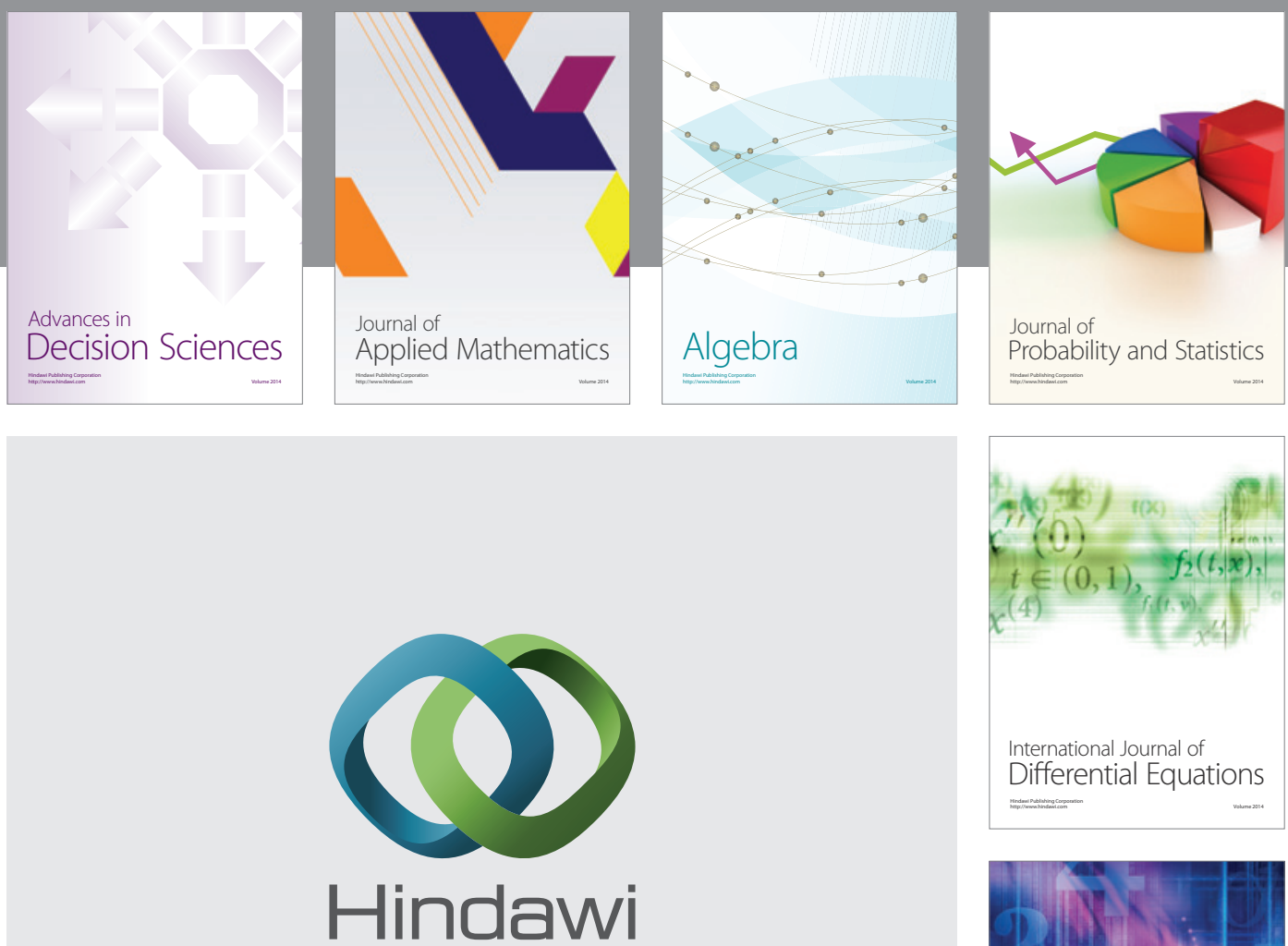

Submit your manuscripts at http://www.hindawi.com
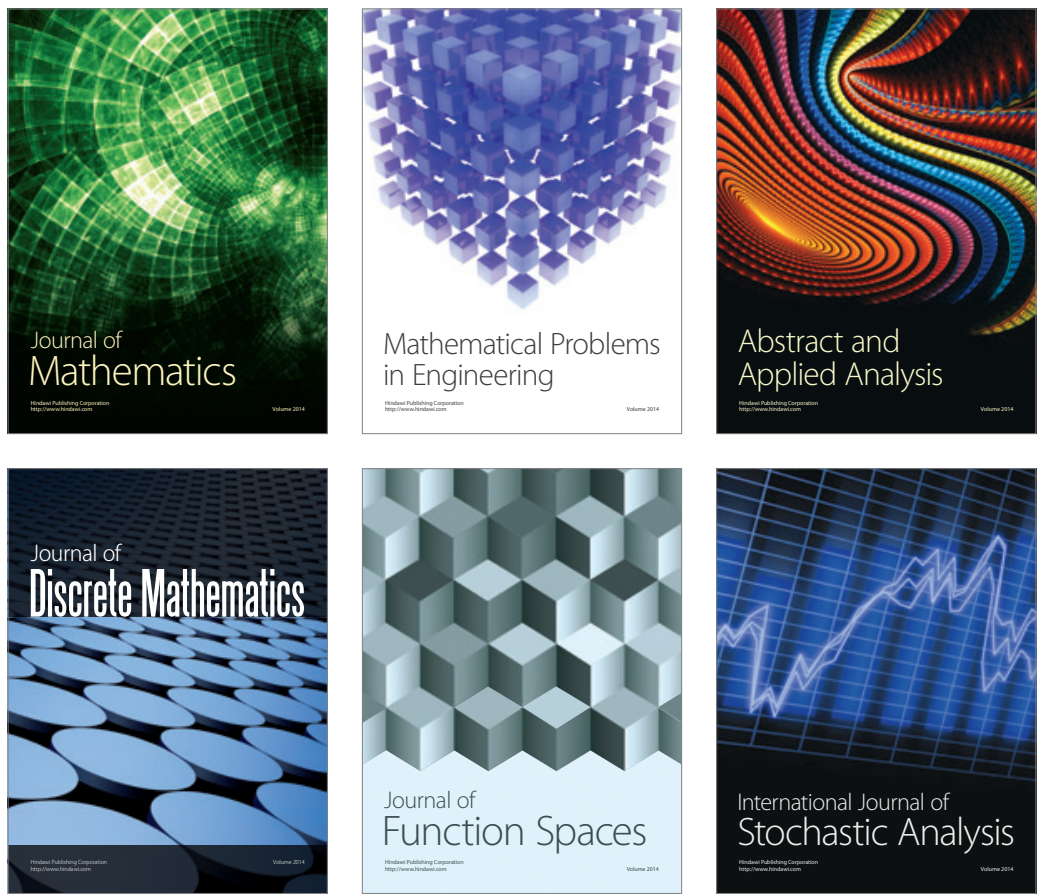

Journal of

Function Spaces

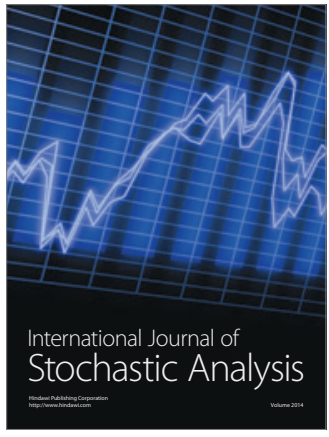

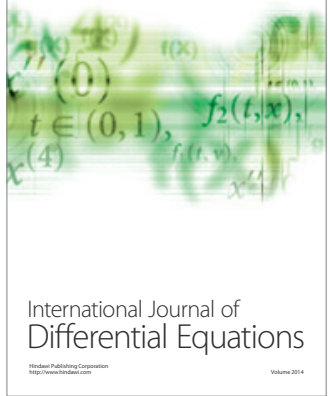
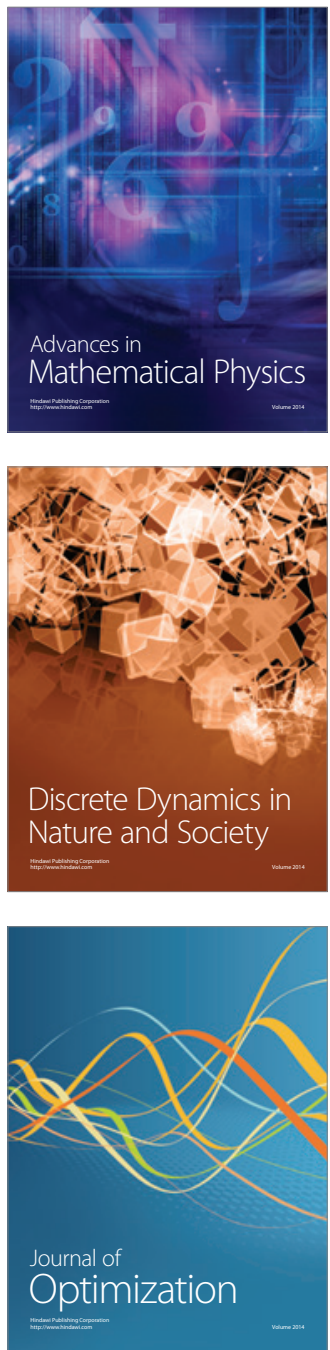